\title{
Allergic inflammation is exacerbated by allergen-induced type 2 innate lymphoid cells in a murine model of allergic rhinitis*
}

Lin Lin, Fei Dai, Jin-Jin Wei, Xin-Yue Tang, Zheng Chen, Guang-Bin Sun

Department of Otorhinolaryngology-Head and Neck Surgery, Huashan Hospital of Fudan University, Shanghai, China
Rhinology 55: 339-347, 2017

https://doi.org/10.4193/Rhino17.065

*Received for publication:

April 5, 2017

Accepted: May 26, 2017

\begin{abstract}
Background: Group 2 innate lymphoid cells (ILC2s) represent a new innate effector leukocyte population that mediates type-2 immune response. However, the contribution of ILC2s to allergic rhinitis (AR) is currently not well defined.
\end{abstract}

Objective: To evaluate the potential existence and function of allergen-induced ILC2s in the experimental AR.

Methods: We established a murine model of AR using ovalbumin (OVA) and aluminium hydroxide. The OVA-induced ILC2s were sorted and purified from the mouse nasal-associated lymphoid tissue (NALT). Then, we assessed ILC2s responses to mouse recombinant interleukin (rmIL)-25, anti-IL17RB antibody and CC chemokine ligand (CCL) 25 in the culture. After that, we adoptively transferred the NALT-derived ILC2s alone or plus rmIL-25 or anti-IL17RB antibody to the murine model of AR to investigate their role in the nasal allergic inflammation.

Results: We showed that ILC2s could be induced by OVA in the NALT of AR model. They were induced to secrete IL-5 and IL-13 by rmIL-25, and blocking of IL17RB contributed to the decreased production of these cytokines in the culture. We found that CCL25 induced the NALT-derived ILC2s migration through CC chemokine receptor 9 on ILC2s in vitro. Numbers of sneezing and nasal rubbing as well as counts of invasive eosinophils were all enhanced after the adoptive transfer of cultured ILC2s in vitro. The expressions of IL-5, IL-13, IL-25 and CCL25 in the NLF of allergic mice were also increased.

Conclusion: These findings show that ILC2s play a proinflammatory role in the murine AR model, and also highlight ILC2s as a new target in the future AR therapy.

Key words: ILC2s, allergic rhinitis, murine, model, NALT

\section{Introduction}

Allergic rhinitis (AR) occurs as a consequence of abnormal immunologic responses to environmental allergens. The disease is defined as symptoms of sneezing, nasal pruritus, nasal blockage, and mostly clear nasal discharge caused by lgE-mediated reactions against inhaled allergens, and is related to the nasal mucosal inflammation driven by type 2 helper T (Th2) cells ${ }^{(1)}$. The activation of Th 2 cells characterizes the type 2 adaptive immune response by producing the cytokines interleukin (IL)-4, $\mathrm{IL}-5$, and IL-13 and inducing B-cell class-switching to $\lg \mathrm{E}^{(2)}$.
The IL-13 gene is expressed in the nasal mucosa of patients with perennial AR or after allergen provocation ${ }^{(3,4)}$. Mice that were sensitized intraperitoneally and challenged intranasally showed the increased level of IL-13 in nasal mucosa compared with challenged-only mice ${ }^{(5)}$. IL-13 is a major contributor to the development of a late nasal response with little influence on the early response, and without affecting nasal eosinophilic inflammation in the experimental $A R^{(6)}$. However, there seems to be a tight correlation between IL- 5 and the number of activated eosinophils in nasal mucosa ${ }^{(7)}$. 
Although Th2 cells are an important source of type 2 cytokines, it is becoming increasingly clear that group 2 innate lymphoid cells (ILC2s) provide another more source of IL-13 and IL-5 ${ }^{(8)}$. Studies also demonstrate that the cytokines IL-25 and IL-33 are important initiators of type 2 responses, and the treatment of mice with IL-25 or IL-33 provokes strong induction of type 2 cytokines and pathology ${ }^{(9,10)}$. Although IL-25 and IL-33 belong to different cytokine families, IL-17 and IL-1, respectively, they lead to similar responses.

ILC2s have been identified as novel IL-13-producing innate cells which are defined as lineage (lymphocyte, macrophage, dendritic cell, basophil, eosinophil, mast cell, and natural killer cell) negative and inducible costimulator (ICOS) positive ${ }^{(11)}$. These cells also express both the IL-25 receptor (IL-17RB) and the IL-33 receptor (T1/ST2) ${ }^{(12)}$. Similar innate Th2 cells, termed natural helper cells, have also been reported ${ }^{(13,14)}$. ILC2s are induced to secrete IL-13 and IL-5 by both IL-25 and IL-33 after Nippostrongylus brasiliensis infection in the gut ${ }^{(11,12)}$. In addition, these cells have been reported to be an essential source of IL-13 for this kind of helminth expulsion, and can migrate into the small intestine, possibly because of their expression of the CC chemokine receptor (CCR) 9. It has been reported that ILC2s are induced by ovalbumin (OVA) as part of the allergic inflammatory lung response, and are the major source of non-T cell-derived IL-13 in the lung ${ }^{(15)}$. However, the contribution of ILC2s to AR is currently not well defined. Because IL-13 is known to be crucial for the late response of the development of $A R$, the investigation of ILC2s is very important.

In this study, we established a murine model of AR with OVA. We showed that the OVA-induced ILC2s from mice nasal-associated lymphoid tissue (NALT) possibly migrated into nasal mucosa of mice, and exacerbated allergic inflammation in the AR model through the production of IL-5 and IL-13 induced by IL- 25 .

\section{Materials and methods}

\section{Animals}

Female BALB/c mice (6-8 weeks old) were purchased from the Chinese Academy of Sciences Shanghai Laboratory Animal Center. These mice were maintained in horizontal laminar flow cabinets and provided sterile food and water in a specific pathogen-free facility. Animal studies were approved by the Institutional Animal Care and Use Committee of Fudan University. The mice were randomly divided into 6 experimental groups ( $\mathrm{n}$ $=12$ for each group).

\section{Allergic model system}

According to published procedures ${ }^{(16)}$, mice were administered $0.5 \mathrm{mg} / \mathrm{ml}$ of OVA (grade V; Sigma-Aldrich, St. Louis, MI, USA) and $20 \mathrm{mg} / \mathrm{ml}$ of aluminium hydroxide (Sinopharm Chemical Reagent Co Ltd., Shanghai, China) in normal saline at a dosage of $0.2 \mathrm{ml}$ per mouse by intraperitoneal injection. The sensitiza-

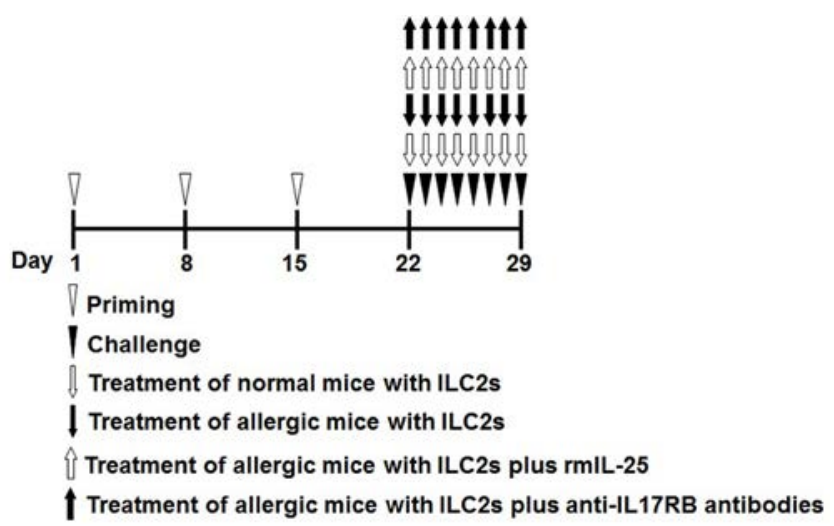

Figure 1. Study protocols. A murine model of allergic rhinitis was prepared by intraperitoneal immunization of ovalbumin on days 1,8 , and 15 (priming) followed by daily intranasal ovalbumin challenge on days 22 to 29 . A group of mice received the challenge treatment of normal saline alone (Normal group), another group of allergic mice was not treated with ILC2s (Control group). Some groups of mice [Treatment group 1: treatment of normal mice with ILC2s, Treatment group 2: treatment of allergic mice with ILC2s, Treatment group 3: treatment of allergic mice with ILC2s plus mouse recombinant (rm) interleukin (IL)25, Treatment group 4: treatment of allergic mice with ILC2s plus antiIL17RB antibody] received the adoptive transfer of ILC2s cultured in vitro alone or plus rmIL-25 or anti-IL17RB antibody intravenously in the tail vein on challenging days, respectively. ILC2S, group 2 innate lymphoid cells.

tion was repeated 3 times at weekly intervals (days 1, 8, and 15). Thereafter, mice were challenged by daily instillation of OVA solution droplet $(40 \mathrm{mg} / \mathrm{ml}$ in normal saline) into the nostrils $(0.02$ $\mathrm{ml}$ per mouse) using a micropipette on days 22 to 29 (Figure 1). As a negative control, a group of mice received the challenge treatment of normal saline alone (Normal group). A group of normal mice (Treatment group 1: treatment of normal mice with ILC2s) or allergic mice [Treatment group 2: treatment of allergic mice with ILC2s, Treatment group 3: treatment of allergic mice with ILC2s plus mouse recombinant ( $\mathrm{rm}$ ) IL-25, Treatment group 4: treatment of allergic mice with ILC2s plus anti-IL17RB antibody] received the adoptive transfer of ILC2s cultured in vitro alone or plus rmIL-25 or anti-IL17RB antibody intravenously in the tail vein on challenging days, respectively, whereas another group of allergic mice was not treated with ILC2s (Control group). Nasal symptoms were evaluated by counting numbers of sneezes and nasal rubs during the 10 minutes immediately after the last OVA intranasal provocation on day 29.

\section{Sample preparation}

After mice were killed, the fore teeth were cut off. The lower jaw and cheek muscles were removed, and NALT was exposed by carefully peeling away the palate. NALT was localized on the posterior part of the plate, and teased out with syringe needles 
in ice-cold RPMI 1640 culture medium supplemented with 10\% FBS. Then the cells were passed through a $70 \mu \mathrm{m}$ Falcon cell strainer. RBC were lysed in Tris-buffered ammonium chloride solution $(0.83 \% \mathrm{NH} 4 \mathrm{Cl}$ and $20 \mathrm{mM}$ Tris/Cl).

\section{Flow cytometry}

NALT cell suspensions were centrifuged for 10 minutes at $200 \times \mathrm{g}$ at $4{ }^{\circ} \mathrm{C}$, and cells were harvested for flow cytometry analysis. Gating strategy for ILC2s was based on CD3CD4CD8CD19CD11bCD11cFceR1 (lineage)-ICOS ${ }^{+}$cell surface expressions ${ }^{(15)}$. The cells were washed with $200 \mu$ l flow cytometry medium [FCM, $1 \%$ BSA and $0.1 \%$ sodium azide in phosphate-buffered saline (PBS)] and incubated with $50 \mu \mathrm{l}$ rabbit serum for 5 minutes at room temperature to block the Fcy receptor. The cells then were washed three times with FCM, and stained with $50 \mu \mathrm{l}$ of monoclonal antibodies against the following surface molecules at $4{ }^{\circ} \mathrm{C}$ for 30 minutes at $1 \times 10^{8}$ cells/ml: CD3, CD4, CD8, CD11b, CD11c, CD19, FcER1, IL17RB; all antibodies purchased from MyBioSource, Inc. (San Diego, CA, USA) or BioLegend (San Diego, CA, USA) and conjugated to fluorescein isothiocyanate, and ICOS and CCR9 monoclonal antibody (both purchased from MyBioSource) were conjugated to Phycoerythrin). They were washed again and incubated with $50 \mu l$ secondary antibody (biotin-conjugated rabbit anti-mouse

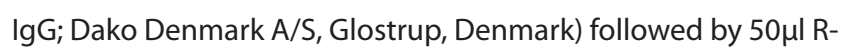
phycoerythrin-conjugated streptavidin (RPE-streptavidin; Dako Denmark A/S). After staining, cells were resuspended in staining buffer containing DAPI for exclusion of dead cells. Controls for single color analysis were carried out by incubating cells with a primary antibody of the same isotype, but of irrelevant specificity at matched concentrations followed by secondary reagents, as described above. The samples were collected on an LSR II flow cytometer equipped with FACSDIVA software (BD Biosciences, Mountain View, CA, USA) and analyzed with FlowJo version 10 (Tree Star, Inc., Ashland, OR, USA). Set-up of the instrument was carried out visually and selection of the isotype control antibody was according to the primary antibodies' host species, isotype, and conjugation format.

\section{ILC2s cell culture}

ILC2s were sorted and purified as CD3CD4CD8CD19CD11b CD11cFceR1 (lineage)-ICOS+ cells at a maximum concentration of $6 \times 10^{7}$ cells $/ \mathrm{ml}$. They were cultured for 6 days in RPMI containing 10\% FCS, $1 \%$ penicillin/streptomycin, $0.1 \%$ $\beta$-mercaptoethanol, $10 \mathrm{ng} / \mathrm{ml}$ of mouse $\mathrm{rmIL}-7$ (BioLegend), and $10 \mathrm{ng} / \mathrm{ml}$ of mouse $\mathrm{rmlL}-33$ (BioLegend).

ILC2s were incubated with $100 \mathrm{ng} / \mathrm{ml}$ of $\mathrm{rmlL}-25$ (BioLegend) or $1000 \mathrm{ng} / \mathrm{ml}$ of anti-IL17RB antibody (MyBioSource) in the presence of rmlL-25 in the cultures ${ }^{(17)}$. The cultures were kept for 3 days. The cell supernatants were used to measure IL-5 and IL-13 production by enzyme-linked immunosorbent assay
(ELISA), and the cell lysates were used to assess the level of CCR9 by ELISA and the messenger (m) RNAs of IL-5, IL-13 and CCR9 by real-time reverse transcription-polymerase chain reaction (RT$P(R)$. Then, ILC2s alone or these cells after treatment with rmIL25 or anti-IL17RB antibody were resuspended in normal saline, and were adoptively transferred at $100 \mathrm{ml}$ per mouse $\left(6 \times 10^{9}\right.$ cells/mouse) intravenously in the tail vein on challenging days.

\section{Transendothelial migration assay}

In accordance with published protocols ${ }^{(18)}$, murine thymic endothelioma cells were cultured in transwell polycarbonate culture inserts placed in 24-well culture plates $(8.0-\mu \mathrm{m}$ pore diameter, BD Falcon, Franklin Lakes, NJ, USA), and allowed to grow to confluence. The above cell monolayers were prestimulated with $40 \mathrm{ng} / \mathrm{ml}$ of mouse rmIL-4 (R\&D Systems, Inc., Minneapolis, MN, USA) for 30 minutes, washed and added to 24-well culture plates containing normal saline or $100 \mathrm{ng} / \mathrm{ml}$ of mouse $\mathrm{rmCCL} 25$ (R\&D Systems). The NALT-derived ILC2s ( $10^{6}$ cells/well) were added to the thymic endothelioma cell monolayers and allowed to migrate for 3 hours $\left(37^{\circ} \mathrm{C}, 5 \% \mathrm{CO}_{2}\right)$. lgG isotypes were used as irrelevant antibodies. Transmigrated cells were counted and stained for flow cytometry, as described above. Results are expressed as transmigration index, generated by using the number of cells that migrated toward normal saline as comparison.

\section{Nasal lavage fluid (NLF)}

After exsanguinations of the mice, one blunted 18-gauge needle was pointed toward the heads of a portion of mice for NLF. One injection of $2000 \mu \mathrm{l}$ normal saline was performed and the fluid was collected with a tube under both nares of the nose for NLF. A portion of NLF was centrifuged for 10 minutes at $150 \mathrm{~g}$ at $4^{\circ} \mathrm{C}$. The supernatants were stored at $-70^{\circ} \mathrm{C}$ for IL-5, IL-13, IL-25 and CCL25 assays. Another portion of NLF was collected for detection of eosinophils, and differential cell counts on 150 cells were performed on cytospins (Cytospin 4 Shandon Ltd., Runcorn, UK) stained with Giemsa ${ }^{(18)}$.

\section{ELISA analysis}

IL-5 in the NLF and the culture was measured using a 96-well microplate coated with $1 \mu \mathrm{g} / \mathrm{ml}$ of IL-5 rabbit antibody. The microplate was incubated in $3 \%$ bovine serum albumin at $37^{\circ} \mathrm{C}$ for 1 hour. Samples at 1:10 dilution were then added followed by incubation at $37^{\circ} \mathrm{C}$ for 1 hour. After washing, anti-IL-5 antibody (R\&D Systems, Inc.) that had been biotinylated using a biotinylation kit (American Qualex International Inc, San Clemente, (A, USA) was then added at $1 \mu \mathrm{g} / \mathrm{ml}$ and allowed to incubate at $25^{\circ} \mathrm{C}$ for 1 hour. After washing, $1.5 \mu \mathrm{g} / \mathrm{ml}$ of streptavidin peroxidase was added followed by incubation at $25^{\circ} \mathrm{C}$ for 1 hour. After washing, tetramethylbenzidine (TMB) substrate $(12.5 \mathrm{~mL}$ of citric-phosphate buffer, $200 \mu$ l of TMB stock solution [ $6 \mathrm{mg} /$ $\mathrm{ml}$ in dimethyl sulfoxide], $100 \mu \mathrm{l}$ of $1 \%$ hydrogen peroxide; Fluka 
Chemical Co, Ronkonkoma, NY, USA) was added to produce a color reaction. The reaction was terminated by the addition of $6 \mathrm{~N}$ sulfuric acid. Optical density was determined at $450 \mathrm{~nm}$ using a microplate reader (Microplate Reader MTP-32; Corona Electric, Ibaraki, Japan). Concentrations of IL-13, IL-25 and CCL25 in the NLF, and IL-13 and CCR9 in the culture was evaluated using corresponding ELISA kits (purchased from R\&D Systems or MyBioSource).

\section{Real-time RT-PCR}

Total RNA of a portion of samples was extracted with Trizol (Invitrogen, Carlsbad, CA, USA) and treated with RNase-free DNase. For reverse transcription, $2 \mu \mathrm{g}$ of above RNA was reversely transcribed with random hexamers (Invitrogen) and cDNA was amplified according to the manufacturer's instructions. Primers were designed using Primer Express software (ABI) from sequence available in Genbank and were synthesized (Geneland Biotech, Shanghai, China). Real-time RT-PCR was performed to detect mRNAs of IL-5, IL-13, and CCR9. IL-5 primers were: forward primer 5'-TCACCGAGCTCTGTTGACAA-3' and reverse primer 5'-CCACACTTCTCTTTTTGGCG-3'. IL-13 primers were: forward primer 5'-AGACCAGACTCCCCTGTGCA-3' and reverse primer 5'-TGGGTCCTGTAGATGGCATTG-3'. CCR9 primers were: forward primer 5'-CCATGACTGACATGTTCCT-3' and reverse primer 5'-TCATAGGGAGAGAGCCCCCGA-3'. GAPDH mRNA was also examined to control the sample-to-sample variation in RNA isolation and integrity by using a pair of primers: forward primer 5'-ACCACAGTCCATGCCATCAC-3' and reverse primer 5'-TCCACCACCCTGTTGCTGTA-3'. After initial denaturation at $95^{\circ} \mathrm{C}$ for 10 minutes, the amplification profile was 15 seconds of denaturation at $95^{\circ} \mathrm{C}, 1$ minute of annealing and extension at $60^{\circ} \mathrm{C}$ for 45 cycles. Negative control RT reaction mixtures contained no reverse transcriptase and no CDNA in the PCR amplification mixtures. For measurement $2 \mu \mathrm{l}$ of diluted CDNA was amplified in a total reaction volume of $20 \mu \mathrm{l}$ by using an 7500 real-time PCR System (Applied Biosystems, Foster City, CA, USA) with $20 \times$ SYBR Green mixture (Invitrogen). Specificity of PCR products was evaluated by melting curve analysis and by size in agarose gels. Using three dilutions of CDNA, linearity of PCR amplification was controlled. Evaluation of data was performed using the $\triangle \mathrm{CT}$ method with GAPDH as internal standard.

\section{Statistical analysis}

Statistical analysis was performed using a commercially available statistical software prism 6.0 (GraphPad Software Inc., San Diego, CA, USA). Data were analyzed with the unpaired Student t test \pm Welch's correction or one-way ANOVA followed by Tukey's multiple comparison test, and presented as mean \pm SEM (standard error of the mean). Significance of a difference was accepted at the $5 \%$ level of confidence. $p<0.05$ was considered statistically significant.
A

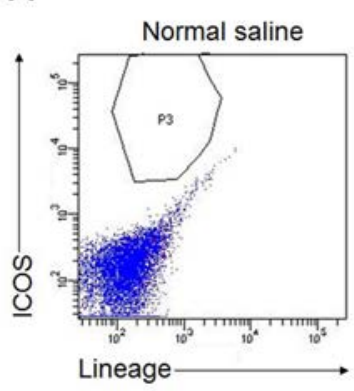

C

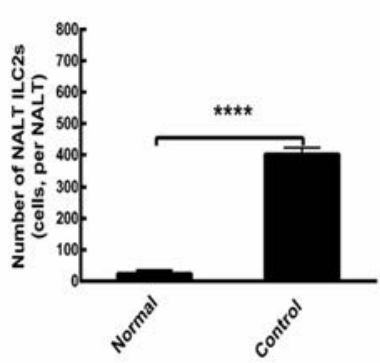

$\mathrm{E}$

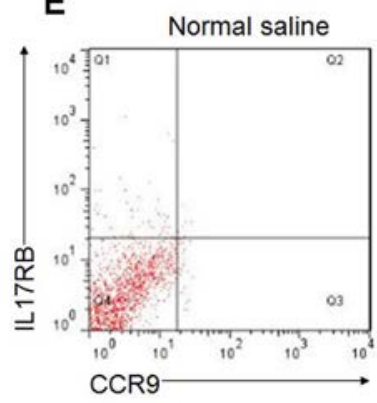

B

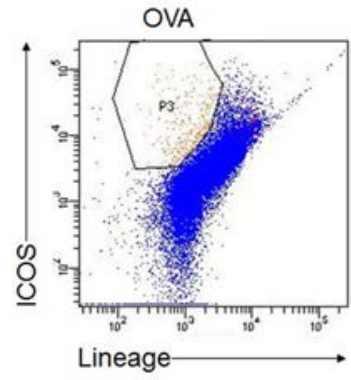

D

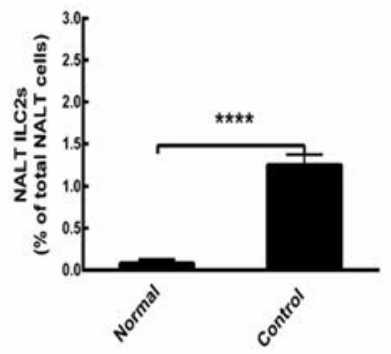

F

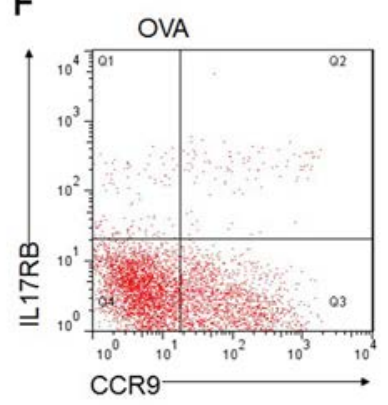

Figure 2. ILC2s derived from nasal-associated lymphoid tissue (NALT) in a murine model of allergic rhinitis. (A) ILC2s were identified as CD3CD4CD8CD19CD11bCD11cFceR1 (lineage)-ICOS ${ }^{+}$cells in normal mice. (B) ILC2s were identified as CD3CD4CD8CD19CD11bCD11cFceR1 (lineage) ${ }^{-}$ICOS $^{+}$cells in allergic mice. (C) Number of the NALT-derived ILC2s. (D) Percentage of the NALT-derived ILC2s. (E) the NALT-derived ILC2s were further defined as IL-17RB ${ }^{+}$CCR9 ${ }^{+}$in normal mice. (F) the NALT-derived ILC2s were further defined as IL-17RB ${ }^{+} C C R 9^{+}$in allergic mice. Each value represents the mean (SEM) of 12 mice in each group. ****p $<0.0001$ vs control group. OVA, ovalbumin. Normal, normal group. Control, control group. ILC2S, group 2 innate lymphoid cells.

\section{Results}

ILC2s arise in NALT during AR inflammation

AR inflammation occurs as a direct consequence of type 2 cytokines release by immune system cells, such as Th2 cells, B cells, mast cells and eosinophils, etc. Mice were sensitized and challenged with OVA to determine whether ILC2s exist in NALT. In this protocol, ILC2s were sorted and purified as lineage-ICOS cells in NALT of mice, and were increased during AR inflammation (Figure 2A and B). Comparisons of ILC2s number and percentage showed that their total number and total percentage all 
A
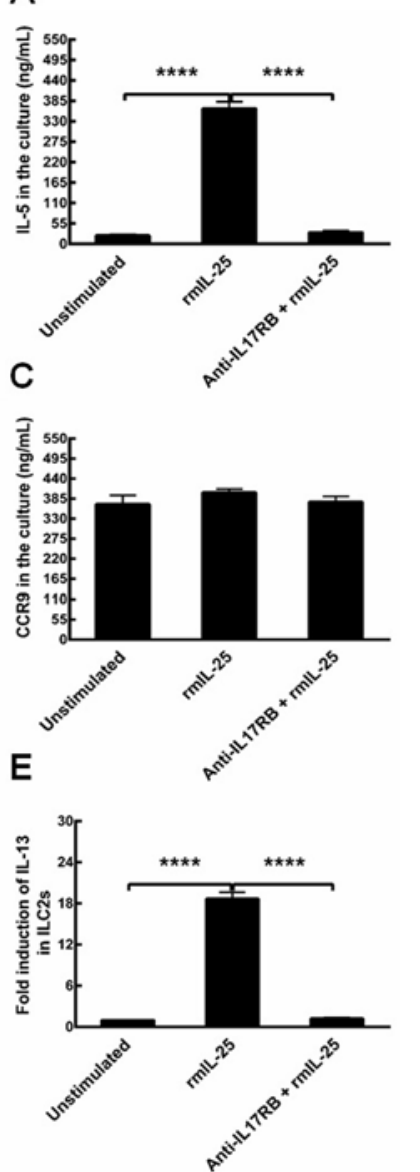

B
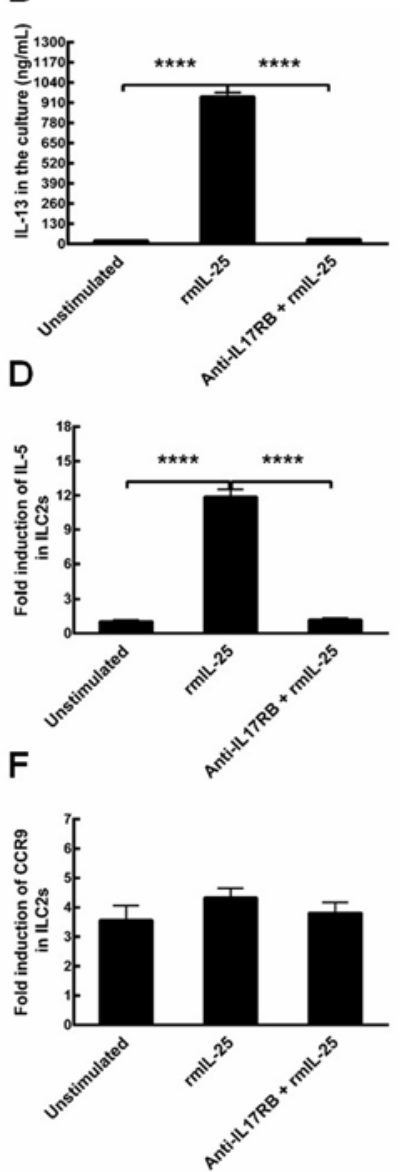

Figure 3. Responses of the nasal-associated lymphoid tissue (NALT)derived ILC2s to mouse recombinant ( $\mathrm{rm}$ ) interleukin (IL)-25 and antiIL17RB antibody in the culture. (A) IL-5 in the culture. (B) IL-13 in the culture. (C) CC chemokine receptor (CCR)9 in the culture. (D) messenger RNA (mRNA) of IL-5 in the culture. (E) mRNA of IL-13 in the culture. (F) mRNA of CCR9 in the culture. Each value represents the mean (SEM) of 12 mice in each group. ${ }^{* * *} \mathrm{p}<0.0001$ vs rmIL-25. Unstimulated, unstimulated samples. rmIL-25, rmIL-25-treated samples. Anti-IL17RB + rmIL-25, anti-IL17RB antibody + rmIL-25-treated samples. ILC2S, group 2 innate lymphoid cells.

increased in NALT in response to OVA treatment compared with those seen in normal samples (Figure 2C and D). IL-17RB and CCR9 were also observed on a proportion of cells already defined as ILC2s, and the number of IL-17RB+CCR9+ ILC2s increased significantly in AR samples (Figure 2E and F).

Type 2 cytokines are induced by rmIL-25 from the NALTderived ILC2s in the culture

To investigate whether the NALT-derived ILC2s secrete type 2 cytokines, we sought to examine the responses of these cells to rmIL-25 in the culture. After ILC2s were stimulated with rmIL-25 or unstimulated, culture supernatants were analyzed by ELISA. IL-5 and IL-13 production were all upregulated in the superna-

tants with rmIL-25 treatment in comparison with the unstimulated control, and the elevation of IL-13 was much higher (Figure $3 A$ and $B$ ). In order to further analyze the induction of type 2 cytokines by rmIL-25, we administered anti-IL-17RB antibody in the culture to investigate ILC2s responses after the blockade of IL-25 receptors in these cells. Cell supernatants were also analyzed by ELISA to quantify cytokine levels. We found that the expressing levels of IL-5 and IL-13 were all downregulated compared to those after the only rmlL-25 treatment (Figure $3 \mathrm{~A}$ and $\mathrm{B}$ ). It has been reported that CCR9 expression can be upregulated by IL-4 ${ }^{(19)}$. However, the contribution of IL-25 to CCR9 in ILC2s is not well defined. In this study, we assessed the production of CCR9 production using ELISA after the administration of rmIL-25 or anti-IL-17RB antibody in the presence of rmIL-25 in the culture. The results indicated that CCR9 was not changed statistically whether in the stimulation of rmIL-25 or anti-IL-17BR antibody state or in the unstimulation state in vitro (Figure $3 C$ ). To further study the secretions of type 2 cytokines induced by rmIL-25 from the NALT-derived ILC2s in the culture, we performed realtime RT-PCR to investigate of the mRNAs of IL-5 and IL-13. The levels of IL-5 and IL-13 mRNA were all increased in ILC2s after rmIL-25 treatment in comparison with the unstimulated control, and the enhancement of IL-13 was significantly higher (Figure $3 \mathrm{D}$ and $\mathrm{E}$ ). The concentrations of them were all reduced after the administration of anti-IL-17RB antibody (Figure 3D and E). In addition, the mRNA of CCR9 was also not changed significantly after the above two treatments compared to that in the unstimulated sample, just like the expression of CCR9 protein.

\section{Migration of the NALT-derived ILC2s is induced by rmCCL25} in vitro

It is evidenced that CCL25 is the unique ligand of CCR9 (20). However, CCL25/CCR9 chemotactic axis is not well understood in ILC2s. CCL25 has been shown to induce lymphocyte adhesion to VCAM-1 and MadCAM-1, and murine thymic endothelioma cells expressed these two molecules whose expression was increased after IL-4 stimulation ${ }^{(21,22)}$. Therefore, we performed transendothelial migration assay using murine thymic endothelioma cells cocultured with rmIL-4 to investigate whether rmCCL25 could induce the migration of NALT-derived ILC2s in vitro. The results are expressed as transmigration index, generated with the number of cells that migrated toward normal saline as comparison. According to the results, rmCCL25 induced ILC2s migration in vitro chemotaxis (Figure 4A and B). ILC2s chemotactic index was higher after rmCCL25 administration in comparison with that in normal saline control (Figure 4C). The results indicated that the NALT-derived ILC2s could migrate in response to the rmCCL25 stimulation.

Allergic inflammation is exacerbated by the OVA-induced ILC2s in a murine model of AR 
A

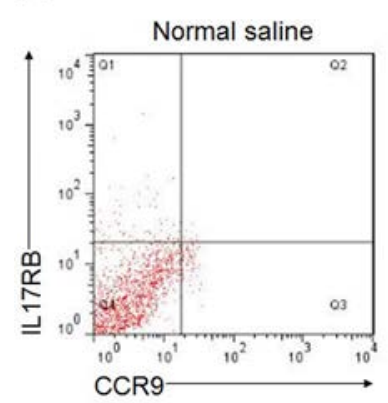

C

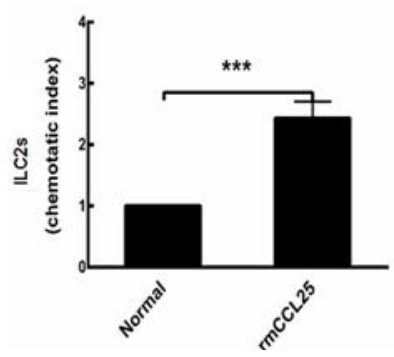

Figure 4. Responses of the nasal-associated lymphoid tissue (NALT)derived ILC2s to recombinant ( $\mathrm{rm}$ ) CC chemokine ligand (CCL) 25 in vitro. (A) Induction of the migration of NALT-derived ILC2s in normal saline-treated samples. (B) Induction of the migration of NALT-derived ILC2s in rmCCL25-treated samples. (C) ILC2s chemotactic index. Each value represents the mean (SEM) of 12 mice in each group. ${ }^{* * *} \mathrm{p}<0.001$ vs rmCCL25. Normal, normal saline-treated samples. rmCCL25, rmCCL25treated samples. ILC2S, group 2 innate lymphoid cells.

To examine the effects of OVA-induced allergic reactions, sneezing and nasal rubbing were counted after the last allergen challenge. AR mice (Control group) showed increases in numbers of sneezing and nasal rubbing compared with those of normal mice (Normal group) that had received only normal saline challenge (Figure $5 \mathrm{~A}$ and $\mathrm{B}$ ). The number of eosinophils in the NLF of AR mice displays an increase compared to that in normal mice (Figure 5C). The results clearly indicated that the murine model of AR was established successfully. The adoptive transfer of OVAinduced ILC2s intravenously in the tail vein increased numbers of sneezing, nasal rubbing, and eosinophils, and concentrations of IL-5, IL-13, CCL25 and IL-25 in the NLF in treatment of allergic mice with ILC2s and treatment of allergic mice with ILC2s plus mouse recombinant ( $\mathrm{rm}$ ) IL-25 compared to those in Control group (Figure 5A, B, C, D, E, F and G). There were statistical differences between treatment of allergic mice with ILC2s and 3 , and the above parameters were elevated much higher in the latter. However, there were no significant differences in all the above parameters between Normal group and treatment of normal mice with ILC2s, that is to say, the adoptive transfer of OVA-induced ILC2s to normal mice did not induce allergic inflammation in nasal mucosa. Additionally, there were no statistical differences
A
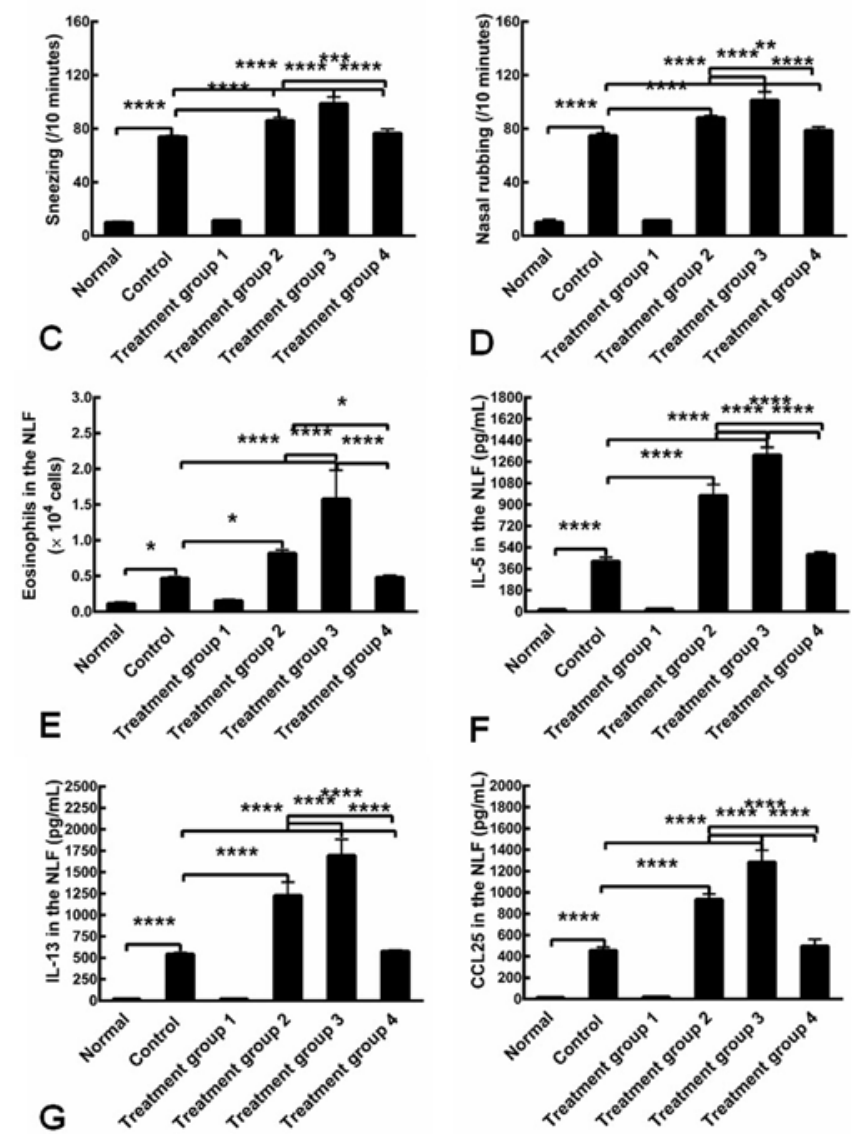

B
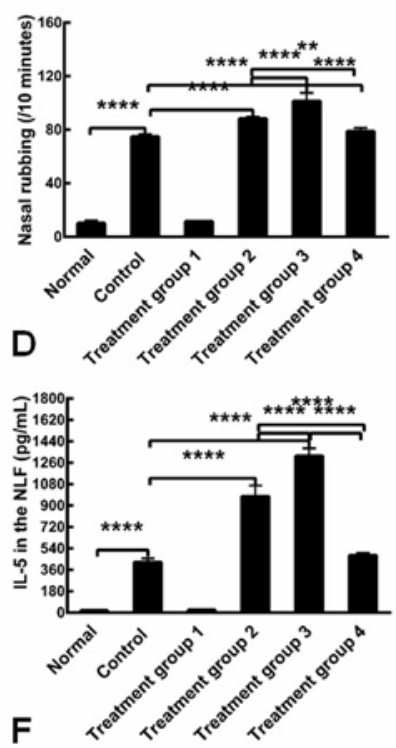

$\mathbf{F}$

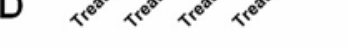

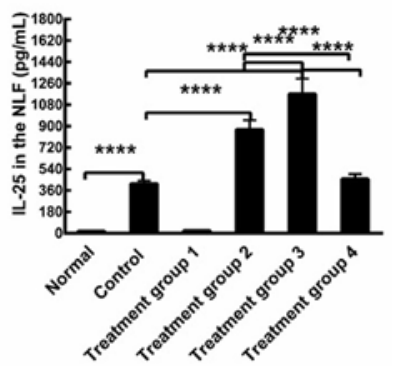

Figure 5. Exacerbation of allergic inflammation by the ovalbumin (OVA)-induced ILC2s in a murine model of allergic rhinitis. (A) Number of sneezing. (B) Number of nasal rubbing. (C) Number of eosinophils. (D) Interleukin (IL)-5 in the nasal lavage fluid (NLF). (E) IL-13 in the NLF. (F) CC chemokine ligand (CCL) 25 in the NLF. (G) IL-25 in the NLF. Each value represents the mean (SEM) of 12 mice in each group. ${ }^{*} \mathrm{p}<0.05$ vs Control group or Treatment group 2. ${ }^{* *} \mathrm{p}<0.01$ vs Treatment group 2. ${ }^{* * *} \mathrm{p}<0.001$ vs Treatment group 2. ${ }^{* * *} \mathrm{p}<0.0001$ vs Control group or Treatment group 2 or Treatment group 3. Normal, normal group. Control, control, group. Treatment group 1, treatment of normal mice with ILC2s. Treatment group 2, treatment of allergic mice with ILC2s. Treatment group 3, treatment of allergic mice with ILC2s plus mouse recombinant (rm) IL-25. Treatment group 4, treatment of allergic mice with ILC2s plus anti-IL17RB antibody. ILC2S, group 2 innate lymphoid cells. 
between treatment of allergic mice with ILC2s plus anti-IL17RB antibody and Control group whether in numbers of sneezes, nasal rubs and eosinophils, or in levels of cytokines in the NLF, in other words, ILC2s migrating into nasal mucosa possibly performed their functions through IL-25 receptor, IL-17RB. These findings demonstrated that ILC2s infiltrating in nasal mucosal of AR mice exacerbated allergic inflammation possibly through the induction of IL- 5 and IL- 13 by IL-25, and the secretion of IL- 25 and CCL25 from epithelial cells (or other types of cells) after the stimulation by ILC2s.

\section{Discussion}

ILC2s represent important new innate cells that produce IL-5 and IL-13 in response to IL-25 and IL-33 that can be produced by airway epithelial cells ${ }^{(10,23)}$. The induction of ILC2s has been demonstrated to be necessary for initiating type 2 responses during Nippostrongylus brasiliensis infection ${ }^{(12)}$, and has been shown to directly induce airway hyperreactivity in the absence of IL-13-producing Th2 cells in allergic lung inflammation ${ }^{(15)}$. Increased numbers of ILC2s were observed in the polyps from CRSwNP patients compared with nasal mucosa from CRSsNP patients or healthy controls ${ }^{(24-28)}$. However, ILC2s alone cannot induce the symptoms of AR including tissue eosinophilia (29), which can be induced solely by activating ILC2s in lungs ${ }^{\left({ }^{30}\right)}$. The role of ILC2s in AR is poorly understood. In this study we aimed to investigate the existence and importance of ILC2s in the type 2 response underlying nasal allergic inflammation.

Our data demonstrated that ILC2s were upregulated in NALT of mice during nasal allergic inflammation. Lineage-ICOS+ ILC2S were detectable in both normal mice and OVA-induced allergic model, indicating that they are present in other lymphoid tissue sites other than mesenteric lymph node. Furthermore, the number of ILC2s increased significantly in the AR model, indicating that these type 2 innate cells might play a role in the development of AR. ILC2s were induced to secrete IL- 5 and IL-13 by IL-25 in the culture, and blocking of IL-25 receptor using anti-IL-17BR antibody prevented the expressions of these two cytokines, which indicated that IL-25 could promote type 2 cytokines secretion from ILC2s not only in vivo ${ }^{(12)}$ but also in vitro. In addition, the mRNAs of IL-5 and IL-13 in ILC2s were also downregulated after blockage of IL-25 signaling pathway in the study. CCR9 is associated with homing of T cells ${ }^{(18)}$, dendritic cells ${ }^{(31)}$, and B cells ${ }^{(32)}$ to the intestinal lamina propria. CCL25/CCR9 axis regulates the inflammatory process during allergic airway inflammation ${ }^{(33)}$. However, this chemotactic axis is not well understood in ILC2s, although it was reported that there was expression of CCR9 on ILC2s ${ }^{(12)}$. CCR9 and its mRNA were also detected in ILC2s cultured in vitro, and their levels were not changed whether with IL-25 treatment or with blocking treatment of anti-IL17RB antibody. The result indicated that CCR9 on
ILC2s could not be influenced by IL-25. Although IL-4 has been shown to drive increased expression of CCR9 on murine T cells when cocultured with dendritic cells ${ }^{(18)}$, the contribution of IL-4 to CCR9 on ILC2s should be further investigated because of the significance of IL-4 in the development of AR. According to our data, CCR9+ ILC2s accumulated in rmCCL25-treated samples more than normal saline samples in transendothelial migration assay, that is to say, rmCCL25 could trigger the migration of CCR9+ ILC2s. CCL25 is constitutively expressed by thymic and small intestinal epithelial cells ${ }^{(34)}$, and is also likely expressed by epithelial cells of lung ${ }^{(35)}$. So we hypothesized that mice nasal epithelia might produce this ligand during allergic inflammation. In the present study, we did not observe the expression of CCL25 in nasal epithelial cells of mice, but the ligand expressing level was definitely upregulated in the NLF of AR mice. Therefore, it was reasonable to speculate that CCL25 produced from nasal mucosa induced the migration of CCR9 ${ }^{+}$ILC2 2 from NALT into nasal mucosa, and these cells performed their functions locally.

Our results demonstrated that the adoptive transfer of NALTderived ILC2s into the murine AR model exacerbated the allergic inflammation. Numbers of sneezing, nasal rubbing and infiltrating eosinophils, and expressing levels of IL-5, IL-13, CCL25 and IL-25 were all enhanced significantly when compared to those of control group. Furthermore, when rmlL-25 was added to the transferred ILC2s, the above parameters were raised more significantly. However, these parameters were reduced when anti-IL-17RB antibody was applied to the adoptively transferred ILC2s. In addition, when the NALT-derived ILC2s were transferred into normal mice, all these factors were not changed. These findings indicated that the OVA-induced ILC2s might aggravate the allergic conditions at least through the secretions of IL- 5 and IL-13 cytokines induced by IL-25.

IL-25 was reported to be expressed in epithelial cells of the murine nasal mucosa after challenge with house dust mites, but not with PBS ${ }^{(36)}$. Although Th2 cells ${ }^{(9)}$, mast cells ${ }^{(37)}$, and natural killer T cells ${ }^{(38)}$ also produce IL-25, it seems that epithelial cells preferentially secrete this cytokine ${ }^{(36)}$. In this current study, we found that the concentration of IL-25 increased in the NLF after the adoptive transfer of ILC2s. In the meantime, the level of CCL25 was also augmented after CCR9+ ILC2s treatment. Recently, CCR9 has been reported to regulate CCL25 expression after OVA stimulation in mice ${ }^{(33)}$. Therefore, there might be a special relationship which is not clearly understood between ILC2s and epithelial cells. Furthermore, these two cell types are all cellular innate defenses, and they all initiate tissue inflammatory responses by producing various cytokines and/or chemokines, which may create a permissive environment for the recruitment of inflammatory cells such as eosinophils and amplification of allergic responses ${ }^{(22,15)}$. 


\section{Conclusion}

In summary, the present study demonstrated that ILC2s arised in NALT during the OVA-induced AR inflammation of mice. Numbers of sneezing, nasal rubbing and invasive eosinophils as well as expressions of IL-5, IL-13, IL-25 and CCL25 in the NLF were all upregulated after the adoptive transfer of the NALT-derived ILC2s. These findings indicated that ILC2s play a proinflammatory role in the murine AR model. It should be emphasized that ILC2s might be a new potential target in the future AR therapy.

\section{Acknowledgements}

This work was supported by the National Natural Science Foun- dation of China (grant no. 81371076), and the Shanghai Suburb Tertiary Hospital Clinical Capacity Building Project (grant no. SHDC12015905).

\section{Authorship contribution}

Conception and design: LL; Analysis and interpretation: LL, FD, JJW, XYT; Drafting the manuscript for important intellectual content: LL, ZC, GBS

\section{Conflict of interest}

The authors have no financial conflicts of interest.

\section{References}

1. Wheatley LM, Togias A. Clinical practice Allergic rhinitis. N Engl J Med. 2015; 372: 456-463.

2. Paul WE, Zhu J. How are $T(H) 2$-type immune responses initiated and amplified? Nat Rev Immunol. 2010; 10: 225-235.

3. Pawankar RU, Okuda M, Hasegawa S, et al. Interleukin-13 expression in the nasa mucosa of perennial allergic rhinitis. Am J Respir Crit Care Med. 1995; 152: 2059-2067.

4. Ghaffar O, Laberge S, Jacobson MR, et al. IL-13 mRNA and immunoreactivity in allergen-induced rhinitis: comparison with IL-4 expression and modulation by topical glucocorticoid therapy. Am J Respir Cell Mol Biol. 1997; 17: 17-24.

5. Miyahara S, Miyahara N, Matsubara S, Takeda K, Gelfand EW. Physiological assessment of allergic rhinitis in mice: role of the high affinity IgE receptor (FceRI). J Allergy Clin Immunol. 2005; 116: 1020-1027.

6. Miyahara S, Miyahara N, Matsubara S, Takeda K, Koya T, Gelfand EW. IL-13 is essential to the late-phase response in allergic rhinitis.J Allergy Clinlmmunol. 2006; 118: 1110-1116.

7. Durham SR, Ying S, Varney VA, et al. Cytokine messenger RNA expression for IL-3, IL-4, IL-5, andgranulocyte/macrophage-colony-stimulating factor in the nasal mucosa after local allergen provocation: relationship to tissue eosinophilia. Immunol. 1992; 148: 2390-2394.

8. Mjösberg J, Bernink J, Golebski K, et al. The transcription factor GATA3 is essential for the function of human type 2 innate lymphoid cells. Immunity. 2012; 37: 649-659.

9. Fort MM, Cheung J, Yen D, et al. IL-25 induces IL-4, IL-5, and IL-13 and Th2-associated pathologies in vivo. Immunity. 2001; 15 985-995.

10. Schmitz J, Owyang A, Oldham E, et al. IL-33, an interleukin-1-like cytokine that signals via the IL-1 receptor-related protein ST2 and induces $\mathrm{T}$ helper type 2-associated cytokines. Immunity. 2005; 23: 479-490.

11. Neill DR, Wong SH, Bellosi A, et al. Nuocytes represent a new innate effector leukocyte that mediates type-2 immunity. Nature.
2010; 464: 1367-1370

12. Neill DR, McKenzie AN. Nuocytes and beyond: new insights into helminth expulsion. Trends Parasitol. 2011; 27: 214-221.

13. Price $A E$, Liang $H E$, Sullivan $B M$, et al Systemically dispersed innate IL-13expressing cells in type 2 immunity. Proc Natl Acad Sci U S A. 2010; 107: 11489-11494.

14. Moro K, Yamada T, Tanabe M, et al. Innate production of $\mathrm{T}(\mathrm{H}) 2$ cytokines by adipose tissue-associated c-Kit(1)Sca-1(1) lymphoid cells. Nature. 2010; 463: 540-544.

15. Barlow JL, Bellosi A, Hardman CS, et al. Innate IL-13-producing nuocytes arise during allergic lung inflammation and contribute to airways hyperreactivity. J Allergy Clin Immunol. 2012; 129: 191-198. e1-4.

16. Hiromura Y, Kishida T, Nakano H, et al. IL-21 administration into the nostril alleviates murine allergic rhinitis. J Immunol. 2007; 179: 7157-7165

17. Ballantyne SJ, Barlow JL, Jolin HE, et al Blocking IL-25 prevents airway hyperresponsiveness in allergic asthma. J Allergy Clin Immunol. 2007; 120: 1324-1331.

18. Costa MF, Bornstein VU, Candéa AL, Henriques-Pons A, Henriques MG, Penido C. CCL25 induces $a_{4} \beta_{7}$ integrin-dependent migration of $\mathrm{IL}-17^{+} \gamma \delta \mathrm{T}$ lymphocytes during an allergic reaction. Eur J Immunol. 2012 42: 1250-1260.

19. Elgueta R, Sepulveda FE, Vilches F, et al Imprinting of CCR9 on CD4 T cells requires IL-4 signaling on mesenteric lymph node dendritic cells. J Immunol. 2008; 180: 65016507

20. Uehara S, Grinberg A, Farber JM, Love PE. A role for CCR9 in T lymphocyte development and migration. J Immunol. 2002; 168 2811-2819.

21. Parmo-Cabañas M, García-Bernal D, GarcíaVerdugo R, Kremer L, Márquez G, Teixidó J. Intracellular signaling required for CCL25stimulated $\mathrm{T}$ cell adhesion mediated by the integrin alpha4beta1. J Leukoc Biol. 2007; 82: 380-391.

22. Miles A, Liaskou E, Eksteen B, Lalor PF Adams DH. CCL25 and CCL28 promote alpha4 beta7-integrin-dependent adhesion of lymphocytes to MAdCAM-1 under shear flow. Am J Physiol Gastrointest Liver Physiol. 2008; 294: G1257-1267.

23. Angkasekwinai $\mathrm{P}$, Park $\mathrm{H}$, Wang $\mathrm{YH}$, et al Interleukin 25 promotes the initiation of proallergic type 2 responses. J Exp Med. 2007: 204: 1509-1517.

24. Shaw JL, Fakhri S, Citardi MJ, et al. IL-33responsive innate lymphoid cells are an important source of IL-13 in chronic rhinosinusitis with nasal polyps. Am J Respir Crit Care Med. 2013; 188: 432-439.

25. Miljkovic D, Bassiouni A, Cooksley C, et al. Association between group 2 innate lymphoid cells enrichment, nasal polyps and allergy in chronic rhinosinusitis. Allergy. 2014; 69: 1154-1161.

26. Walford HH, Lund SJ, Baum RE, et al. Increased ILC2s in the eosinophilic nasal polyp endotype are associated with corticosteroid responsiveness. Clin Immunol. 2014; 155: 126-135.

27. Ho J, Bailey M, Zaunders J, et al. Cellular comparison of sinus mucosa vs polyp tissue from a single sinus cavity in chronic rhinosinusitis. Int Forum Allergy Rhinol. 2014; 5: $14-27$.

28. Ho J, Bailey M, Zaunders J, et al. Group 2 innate lymphoid cells (ILC2s) are increased in chronic rhinosinusitis with nasal polyps or eosinophilia. Clin Exp Allergy. 2015; 45: 394-403.

29. Kato Y, Akasaki S, Muto-Haenuki Y, Fujieda S, Matsushita K, Yoshimoto T. Nasal sensitization with ragweed pollen induces localallergic-rhinitis-like symptoms in mice. PLoS One. 2014; 9: e103540.

30. Yasuda K, Muto T, Kawagoe T, et al. Contribution of IL-33-activated type II innate lymphoid cells to pulmonary eosinophilia in intestinal nematode-infected mice. Proc Natl Acad Sci U S A. 2012; 109: 34513456.

31. Wendland M, Czeloth N, Mach N, et al. CCR9 is a homing receptor for plasmacytoid dendritic cells to the small intestine. CCR9 is a homing receptor for plasmacytoid dendritic cells to the small intestine. Proc Natl Acad Sci U S A. 2007; 104: 6347-6352.

32. Pabst $\mathrm{O}, \mathrm{Oh} / \mathrm{L}$, Wendland $\mathrm{M}$, et al. Chemokine receptor CCR9 contributes to 
the localization of plasma cells to the smal intestine. J Exp Med. 2004; 199: 411-416.

33. López-Pacheco C, Soldevila G, Du Pont G, Hernández-Pando R, García-Zepeda EA. CCR9 Is a Key Regulator of Early Phases of Allergic Airway Inflammation. Mediators Inflamm. 2016; 2016: 3635809.

34. Vicari AP, Figueroa DJ, Hedrick JA, et al. TECK: a novel CC chemokine specifically expressed by thymic dendritic cells and potentially involved in T cell development. Immunity. 1997; 7: 291-301.

35. Ericsson A, Kotarsky K, Svensson M, Sigvardsson M, Agace W. Functional characterization of the CCL25 promoter in small intestinal epithelial cells suggests a regulatory role for caudal-related homeobox (Cdx) transcription factors. J Immunol. 2006; 176: 3642-3651.

36. Nakanishi W, Yamaguchi S, Matsuda A, et al. IL-33, but not IL-25, is crucial for the development of house dust mite antigeninduced allergic rhinitis. PLoS One. 2013; 8 : e78099.

37. Ikeda K, Nakajima H, Suzuki K, et al. Mast cells produce interleukin-25 upon Fc epsiIon Rl-mediated activation. Blood. 2003; 101: 3594-3596.

38. Terashima A, Watarai $H$, Inoue $S$, et al. A novel subset of mouse NKT cells bearing the $\mathrm{IL}-17$ receptor $\mathrm{B}$ responds to $\mathrm{IL}-25$ and contributes to airway hyperreactivity. J Exp Med. 2008; 205: 2727-2733.
Lin Lin

Department of Otorhinolaryngology

Head and Neck Surgery

Huashan Hospital of Fudan University

No. 12 Wulumuqi Middle Road

Shanghai, 200040,

China

Tel: 862152889999

Fax: 862162489191

E-mail: linlinhsn@aliyun.com 